\title{
A Scientific Approach and Perception for Clinical Practice in India
}

\author{
Arti Swami*, Shalaka Abhyankar, Mugdha Kulkarni, Harshal Mundada \\ School of Pharmacy, Dr. Vishwanath Karad MIT World Peace University, Pune, Maharashtra, INDIA.
}

\begin{abstract}
The rate and extent of absorption of a drug from its dosage form is referred to as bioavailability. While bioequivalence between two drug products is attained if their extent and rate of absorption do not relatively differ when administered at the same dose. A bioequivalence study comprises of two or more bioavailability studies which are performed by i) Measurement of active drug substance or its metabolites in the biological fluid; ii) Comparative pharmacodynamic studies in humans; iii) Comparative clinical trials; iv) Comparative in-vitro dissolution studies. The rationale of the study is the monitoring of the pharmacokinetic-pharmacodynamic parameters (AUC, $\mathrm{C}_{\text {max }}$ ) after administration of the tested drug. This study is not an experimental study but a form of data analysis and reporting of comparative bioavailability studies. The study data obtained is required to be equipped with applications of new drug products as notified in Schedule Y. Cumulatively, it aids in the development of pharmaceutical preparations in the pharmaceutical industry. This review article aims to illustrate the systematic overview of $B A / B E$
\end{abstract}

study with an emphasis on its experimental aspects like study design, volunteer selection, dosing regimen, sample collection procedures along with a detailed insight on Informed Consent Document, Case Report Form, Trial Master file and the Protocol which collectively form the backbone of the clinical study.

Key words: Bioavailability, Bioequivalence, Clinical research, Protocol, Case report form, Trial master file.

\section{Correspondence}

Arti Swami,

School of Pharmacy, Dr. Vishwanath Karad MIT World Peace University, 124, Ex-serviceman Colony, Paud Road, Kothrud, Pune-411038, Maharashtra, INDIA. Email id: arti.swami@mitwpu.edu.in

DOI: $10.5530 /$ ijpi.2021.3.44

\section{INTRODUCTION}

Clinical research is an organised study performed on human candidates to collect all the necessary data required for the discovery of new drug products or verification of clinical, pharmacological (pharmacokinetic/ pharmacodynamic) or adverse effects of API/formulations with the aim of determining the ADME profile of drugs. ${ }^{1}$ The basis of clinical research is chiefly the scientific practice of analysing all data procured from the chemical or physical investigations along with accurate instrumentation to record observations, hypothesize and experiment. ${ }^{2}$ Clinical research can be of varied types including treatment, preventive, diagnostic, epidemiological or genetic clinical studies. ${ }^{3}$ It includes phase 1 (Human pharmacology) and 2 (Therapeutic exploratory) which typically differ in the number of volunteers participating. On the basis of the duration of a typical clinical study, it can be classified as pilot (shorter duration) and pivotal (longer duration) clinical study. ${ }^{4}$ The duration of a study is determined by the number of 'periods' the study comprises. A period is defined as a sub-part of a phase of clinical trial, which includes single dosing followed by periodical sampling and observational time span to monitor the occurrence of adverse events/serious adverse events in the subject and lasts usually for 48 to $72 \mathrm{hr}$. Two periods of a single phase are separated by a washout period or a gap which is basically to allow the drug to get completely eliminated from the body, whose duration differs as per the half-life of the drug.

\section{HISTORY AND DEVELOPMENT OF CLINICAL RESEARCH IN INDIA}

During the British era, a large number of drug shipments were imported from overseas but it resulted in heavy inflow of substandard, spurious and adulterated drugs. In response to the increasing demand from the public to regulate the incoming drug supply in India, the Government instated a Drug Enquiry Committee (DEC) $)^{5}$ who introduced the Drug
Bill which, after many revisions came to be known as Drugs and Cosmetics Act, 1940 (D\&C).$^{6-8}$ The Act regulated the manufacture, distribution, import, export and sale of drugs thus becoming the central mandate in drug regulation. The Central Drugs Standard Control Organisation (CDSCO), Drug Technical Advisory Board (DTAB), Drug Consultative Committee (DCC) and Drug Controller General of India (DCGI) were also established under it. ${ }^{9,10}$ The formation of these vast groups of regulatory bodies ultimately facilitated the drug manufacturing sector in India.

The Indian Patent Act (1970) proved to be the factor that boosted generic growth as it gave more importance to the process of manufacturing rather than the novel drug product. ${ }^{11}$ But it was during the TRIPS agreement that India signed when the prospective redrafted Indian Patent Act (2005) was approved which restored the privileges of the product rather than the process of development. The area of clinical research has now gained relevance. ${ }^{12}$ On realizing the importance of clinical research, many amendments were made in the Schedule $\mathrm{Y}^{13}$ of $\mathrm{D} \& \mathrm{C}$ act which now completely encompasses the guidelines and requirements necessary for clinical trials. The growing public demand for foreign products had increased considerably which cleared the path of the foreign industries to set a foot on the Indian land and captured a huge market which affected the sales of Indian products. The Schedule $\mathrm{Y}$ was initially drafted for the generic products, but their increasing interference led to its redrafting in order to regulate their manufacturing. ${ }^{12}$

Due to the lack of proper guidelines and regulations for conducting clinical trials, malpractices were rampant, which finally resulted in formation of ethics committees as well as development of regulatory guidelines. ${ }^{14}$ Ethics committee is a body which comprises of medical, non-medical, scientific and non-scientific members whose main responsibilities include: 
- To guarantee protection of rights, safety and well-being of human subjects involved in clinical trials.

- To oversee drafting and approval of protocol

- To check the suitability of investigators, facilities and methods.

- To verify the adequacy of information to be used for obtaining.

- Documenting informed consent of study subjects and adequacy of confidentiality ${ }^{15,16}$

In addition, Indian Council Medical Research (ICMR) also issued ethical guidelines for biomedical research on human subjects in 2000, closely followed by the Indian Good Clinical Practice (GCP) ${ }^{17}$ by CDSCO in $2001 .{ }^{18}$ In the following years, Indian clinical research for Investigational New Drug (IND), unfortunately, witnessed a lag phase in which many leading companies did abandon drug development due to many factors like large investment costs, lack of necessary skill sets and multiple failures. But in the recent era, India is consciously progressing towards proprietary drug development with a major contribution from the biotechnological industry and increasing registration of independent Contract Research Organisations (CROs) for clinical research practice. ${ }^{11,19}$ Every clinical study can only be conducted after written approval from the Independent Ethics Committee (IEC) ${ }^{20}$ and DCGI. CDSCO has also set registration criteria for CROs with validity up to 5 years which is then subject to re-registration (renewal) for further conduction of clinical trials.

Currently, clinical trials are regulated by:

1) Rule $122 \mathrm{~A}$ through $\mathrm{E}$ of $\mathrm{D} \& \mathrm{C}$ act

2) Schedule $Y$ of D\&C act

3) GCP guidelines

4) Ethical guidelines for biomedical research on human subjects by ICMR.

\section{OVERVIEW OF METHODOLOGY IN BA-BE STUDIES}

The complete flow of the process is as described in [Figure 1]

Step I: Registration and Enrolment of volunteers

Registration of the new volunteers is the first step in a typical clinical study. It is a voluntary process carried out in the facility itself prior to the commencement of the trial. The candidates need to carry their authentic identification proofs with them during registration. All the data of the candidate is uploaded on a centralised software by registration personnel which includes their personal information along with their Body-mass Index (BMI) as they form a critical parameter in volunteer selection for a particular study, language preference, their contact information, their personal habits in relation to diet preferences, consumption of alcohol, smoking and tobacco, X-Ray details, photo, scanned copies of their identification proofs and fingerprint scans as a source of verification in future.

\section{Inclusion criteria for registration}

As the bioequivalence studies ${ }^{21-26}$ are predominantly conducted in healthy volunteers, the candidate desiring to be a volunteer must be healthy in all aspects. He must fit the age criteria required for the study along with having the necessary BMI. In addition, the candidate must be able to read or write either in English or the local language to thoroughly understand the study process and signing of consent.

\section{Exclusion criteria during registration}

If the candidate is found to be unhealthy in any aspect or is an alcoholic or smoker or regular consumer of tobacco then he is deemed unfit to be selected for the study. Also, if the candidate is illiterate, he must bring a LAR (Legally Accepted Representative) with him or must sign the

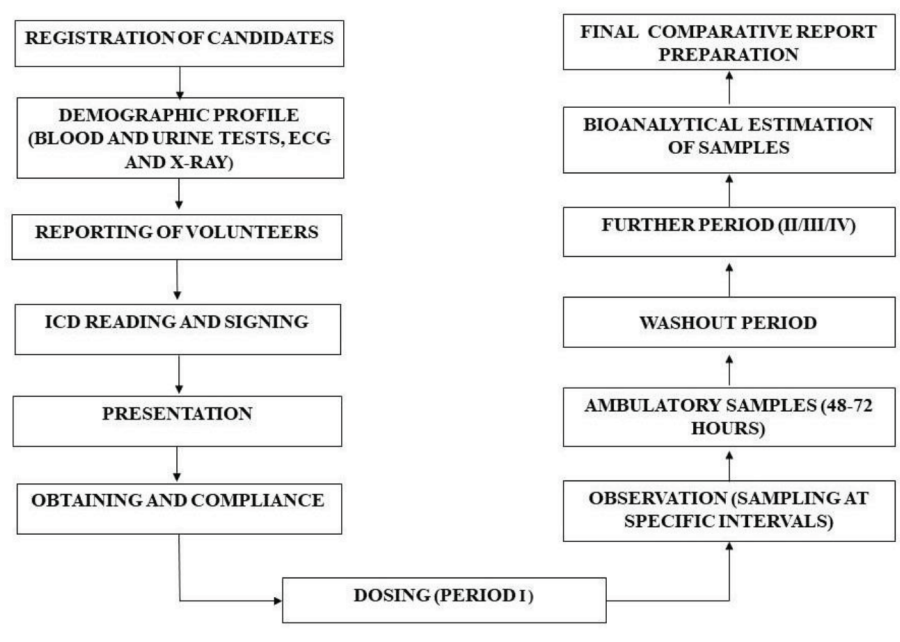

Figure 1: General flow of a clinical research study.

consent in presence of an Impartial Witness (IW) otherwise he will be disqualified from the registration process.

After successful registration, the volunteers are assigned a permanent Volunteer Identification Number (VIN) through the software which will be used for identification purposes in future studies.

Step II: Screening and Medical Examination

Screening and medical examination is performed for all volunteers before every period of every study they wish to appear for. Screening process involves a re-verification of the identity of volunteers. Their fingerprints and assigned volunteer identification numbers (VIN) are crosschecked in the software along with the details of the last study they had participated in. A screening consent needs to be signed by the volunteer which describes all the aspects of the screening process along with the medical tests that need to be performed (Haematological, serological, biochemical tests, HIV test and $\beta$-hCG test for females).

\section{Exclusion criteria for screening}

According to the protocol, the volunteer must have not participated in any clinical study for 90 days before appearing for a screening. This data could be cross checked by the fingerprint verification, which gives the details about the eligibility of volunteers for any type of clinical study.

In medical examination, the volunteers need to undergo basic physiological tests like blood and urine tests, ECG, X-ray and HIV test to check if they are medically fit for study. Questioning of the volunteer for substance and alcohol use is also carried out. Usually, blood sample collection and ECG test is carried out in the facility itself by pathologists and sent for testing to the laboratory mentioned in the protocol, if necessary. For female volunteers, additional details of obstetrics and gynaecological history are also noted. If no deviation is proven by the reports, then the volunteer is considered eligible to be the part of the study.

\section{HIV test and counselling}

In pre-test counselling, the volunteer is well informed about AIDS/HIV and necessary precautions against it.

In post-test counselling, the volunteer is informed about his results and measures to be taken in future. If the test is positive, $100 \%$ confidentiality is assured, a separate counselling is suggested but with permanent blockage of the volunteer on the software.

After passing the medical examination, the volunteers are given an ID card which bears their VIN and the subject number is assigned to them 
for that specific study. It should also be noted that screening and medical examination is usually valid for 21 days after conduction.

Step III: Check-in for trial into the facility

1. Reporting: On the day of check-in for every period, the selected volunteers need to report into the facility where their data is re-verified (fingerprint scanning). In cases where a volunteer appears for a reporting of a study after a few days of initial screening, he has to undergo some re-tests as conducted under medical examination to update the reports.

2. After completion of the reporting process, distribution of Informed Consent Document (ICD) in volunteer's preferred language is carried out. They are provided with ample time to go through the same. An audio-video monitored presentation is organised for all the volunteers after the reading to clear their queries. Only after the same, an individual audio-video monitored written consent (Obtaining) which also is a part of ICD, is taken from the volunteers and is considered as their final consent for being a part of the study. A compliance which is a comprehensive questionnaire; is also procured from them following the obtaining process.

3. Informed consent document

It is a brief document used to ensure everyone involved in clinical research is participating on their own accord, with full knowledge of potential risks and benefits to the extent that it is possible.

Through the Informed consent, the volunteers are made known about

- An explanation about the design (fast/fed states), purpose, procedure (detailed time table of events) and duration of the research.

- Details about the active ingredient with the recommended dose

- Information regarding predictable risks and discomforts as well as possible benefits which can be expected from the research.

- A description stating the confidentiality of the information collected during the trial.

- The availability of a fully-equipped Intensive Care Unit within the facility in case of emergencies.

- Compensation which will be paid for by the sponsor if any injury or serious adverse event occurs along with the entitled monetary compensation that the volunteer will receive if he wishes to withdraw from an ongoing study.

An investigator should only get consent from a research subject if:

- Enough time was given to the subject for considering his participation in the study.

- Persuasion of the research subject is not carried out in any way. ${ }^{27}$

4. Dosing and sample collection

After completing the obtaining process, the volunteers are assigned bed numbers, provided with gowning and a utility kit for their stay. A minimum $8 \mathrm{hr}$ stay in the facility before actual dosing is mandatory along with all the required restrictions on diet and water. ${ }^{28}$ Dosing procedure follows randomisation as mentioned in study designs. Randomisation is simply, a random allotment of test and reference samples to the volunteers to avoid selection bias in a study. ${ }^{29,30}$ A pre-dose blood sample (quantity as mentioned in protocol) is taken $1 \mathrm{hr}$ prior to dosing.

Dosing procedure is done according to trial requirements of the protocol. In case of oral dosage forms, a specified quantity of water is co-administered to achieve dosing uniformity amongst all the subjects. In addition, a sitting posture has to be maintained after the dosing.

Dosing is followed with blood sampling after specific time intervals as mentioned in the protocol. This process generally takes up to $48 \mathrm{hr}$. Occurrence of any adverse effects is monitored regularly. A record of their vital signs during the whole course of sampling is also maintained.
Periodically collected samples need to be immediately stored into a deep freezer in the form of two aliquots until they need to be dispatched to the bioanalytical laboratory for further analysis.

After completion of sampling, the volunteers are allowed to leave the facility after basic check-out tests are performed.

\section{Case Report Form}

The Case Report Form (CRF) is an all-inclusive document which summarizes all the necessary data that is recorded from check-in of the volunteer till the check-out procedures. It includes a comprehensive questionnaire for the volunteer which is duly filled by the in-charge during the compliance process. The vital signs and clinical data recorded during check-in, allotment of beds and lockers, a dosing log which briefs about the products that will be used and restrictions for the volunteer during the process. This report also includes check- out data of the tests performed similar to the ones carried during check-in.

While designing a CRF, recurring data such as protocol ID, code of the site, subject number and volunteer initials are mentioned from the first page till the end thus avoiding duplication of any of its pages.

It is the responsibility of the investigators to fill the case report form promptly during the trial. CRF forms an integral part of the Trial Master File, a document which is submitted to the sponsor after the end of the study. ${ }^{31}$

\section{Trial Master File}

The Trial Master File (TMF) comprises of all vital documents which facilitate the conduction, replication and evaluation of a clinical trial. It is a compendium encompassing the complete details of the clinical trial. ${ }^{32}$

The ICH GCP Section 8 gives an insight on the structural composition of TMF.

The whole document is divided into 3 parts:

- Before clinical trial phase: This section will include the following data; Investigator's brochure, qualification document of the investigator, documents approved by IRB/IEC and Regulatory Authority, Financial aspects of the trial, Insurance statements, signed agreement between the involved parties, signed protocol and CRF copy, Informed Consent Form, Master randomisation list, Decoding procedure for blinded trials, Trial initiation monitoring report, Normal ranges for medical or laboratory tests, Sample of label attached to the product container, instructions for handling of product and its shipping records.

- During the clinical trial phase: This section comprises of- Revision of earlier mentioned documents (if any), signed, dated and completed CRF, Subject ID code and screening log.

- After termination of the clinical trial phase: This section must include following data- Investigational product accountability at the site, Final trial close out monitoring report, Final report to IRB/ IEC by investigator, completed subject ID code list, Documentation of Investigational product destruction, Treatment allocation and decoding documentation in case of blind trial design, Final Clinical study report, audit certificate. ${ }^{33}$

Access and Storage of Trial Master File (TMF): The TMF should be complete and legible throughout the trial. There should be availability of adequate and suitable place for storage and archival of all essential documents so as to prevent them from any physical damage and this place is approved by the sponsor only after conduction of an audit. If the Institution makes a change in arrangement of TMF then it should be notified to Sponsor. The permission to access the document is given by the Sponsor and/or the Institute. Sponsor and/or institute has the authority for any decisions concerning the TMF. 
Quality of TMF: Article 57 of Regulation states that "The Clinical Trial Master File shall at all times contain the essential Document." All information should be filled appropriately in TMF and must be maintained up-to-date. Sponsor should also undertake routine QA measures and ensure availability and accessibility of the TMF to the competent authority. ${ }^{34}$

\section{Quality Management}

It is an integrated system within any institution whose aim is complete quality emulation of the standards as specified in the guidelines of ICH GCP. Quality Control and Quality Assurance are two sub-branches-

1. Quality control: According to GMP and ISO 9000, it is a part of the quality management system which focuses on fulfilling the quality requirements in the study process. Each sub-department has its own personnel responsible for the in-process quality control whose main duty includes systematic, real-time compliance checks in each step of the study process (in-process quality control) and assuring adherence to the protocol procedures.

2. Quality Assurance: Quality Assurance is the final authority in quality management and it validates the effectiveness of the Quality Control and is even responsible for scheduled audit and validation of the facility.

\section{Clinical Trial Protocol}

It is a document that describes the details about conduction of a clinical trial which includes the objective, methodology, design, statistical processing and management of a clinical trial. Protocol also provides assurance about the safety of the subject along with the data integrity. The protocol serves as a guideline for the investigators for a smooth conduction of a trial. It is directed by a clinical researcher but the actual writing is a cumulative effort of a medical specialist, a statistical and pharmacokinetic expert, the clinical research coordinator and the project manager who provide all the input to a medical writer to compile the final document. ${ }^{35}$

ICH Good Clinical Practice guidelines states that a protocol must consist of the following topics:

- Title Page

Details about the $\mathrm{PI} / \mathrm{CI} /$ coordinator/sponsor and abstract of the study.

- Background information

Includes study hypothesis, significance of study, discussion of relevant literature and data.

- Objectives of the study

Includes statement of purpose.

- Study design

Duration of study, subject participation, outcomes to be measured.

- Inclusion and exclusion criteria of subjects

Description of recruitment

- Treatment of subjects

Housing, Diet, Dosing, Sample collection procedure and schedule, total blood loss.

- Assessment of efficacy and safety

Recording of vital signs and examination

- $\quad$ Adverse events

Identification and reporting of AE and SAE.

- Instances of discontinuation of the study

Termination issued by PI/CI or project in-charge, multiple voluntary withdrawals or reports of AE/ SAE.

- $\quad$ Statistics
Assessment of pharmacokinetic parameters, statistical Analysis of Variance (ANOVA)

- Quality control and quality assurance

Details of steps to be taken to assure that the data collected is accurate, reliable and complete, third party monitoring if any.

- $\quad$ Ethics

Institutions responsible for ethical regulation

- Data handling and record keeping

Procedure for subject confidentiality, data storage and protection, record retention as per the sponsor's requirement

- Publication policy

Description of plans for publication of research results

- $\quad$ Project timetable

As stated in Annexure 1 and 2

- References

- Appendices and amendments ${ }^{36,37}$

\section{Bioanalytical estimation}

After complete conduction of the study, the further processing is carried out by the bioanalytical department whose tasks include isolation of plasma from the blood sample and drug extraction by three preliminary methods:

1) Solid phase extraction method (SPE): In this technique separation of desired constituent from a solid sample is achieved by extraction with an organic solvent in which the solubility of other substances present in the sample is negligible. In this technique, hydrophobic functional groups are chemically bonded to a solid surface (eg: powdered silica), these groups will interact with hydrophobic organic compounds by van der waals forces of interaction and extract them from the aqueous sample. Usually, the same solid phase used in chromatographic technique (HPLC) is used for solid phase extraction.

2) Liquid liquid phase extraction (LLE): It is a technique in which a substance dissolved in one phase (aqueous phase) is completely transferred to the second phase (organic phase) which should be essentially immiscible with the first phase. It can be used for extraction of acidic and basic drugs with high extraction recovery.

3) Protein precipitation type of extraction: It is a method used when there is a high amount of analyte present in the plasma sample and is induced by addition of an organic solvent like acetonitrile or methanol, metal ions or salt which act as precipitating agent. It can be followed by SPE or LLE to achieve more efficiency. ${ }^{38}$

On extraction, the analyte is then subjected to advanced chromatographic techniques like GC-MS or LC-MS depending on its specific requirement. ${ }^{39}$ The retrieved data from the analysis is then sent for bio-statistical comparison with the reference product. It is carried out with the help of statistical software like SAS and a final bioequivalence report is prepared.

Randomised dosing (crossover design) in a typical two period study is depicted in Figure 2.

\section{SCOPE AND FUTURE PROSPECTS}

Pharmaceutical and clinical sector has received a tremendous boost owing to COVID-19 pandemic which has created healthy competition amongst the key pharmaceutical companies and has also resulted in setting up of many new industries and research organisations. Clinical sector, especially is bound to expand which will in turn increase investment and requirement of manpower, leading to many job opportunities. Clinical research and trials will be more open and transparent in future which will aid the society to understand and interact with the professionals 


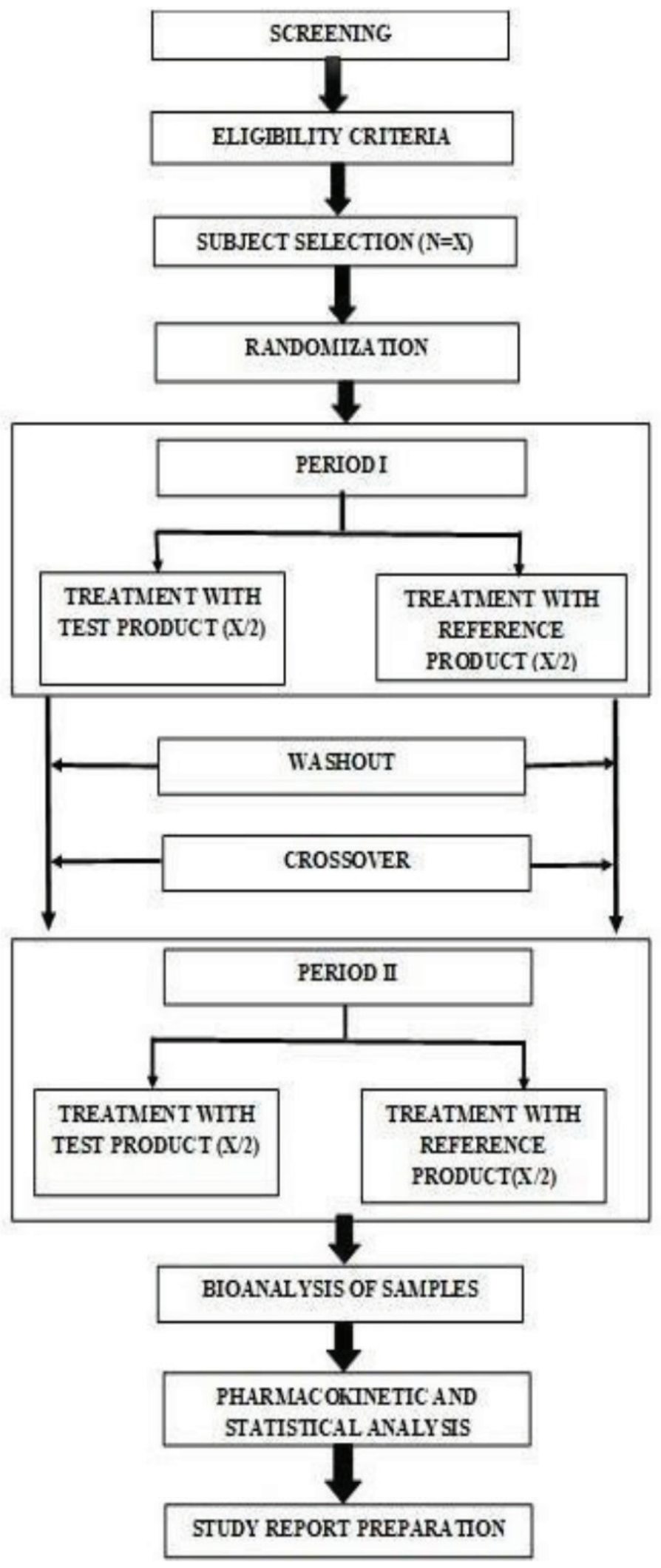

Figure 2: A Typical Two Period Randomised Study.

in the sector. Advancements are in progress to make clinical research more integrated with healthcare and not be looked upon as solely a part of the drug development process. In addition, collaborative and joint efforts over geographical boundaries will allow interaction and rich exchange of ideas, which will aid the evolution of clinical research. The study processes will be more human centered with more emphasis on personalised care of all the participants which can further encourage them to promote participation in trials as past benefactors..$^{40,41}$

\section{CONCLUSION}

This review aims to give a detailed overview of the BA/BE studies conducted at research facilities along with detailed process predominantly involved in clinical research. All the studies are performed according to rigid protocols. Complex bioanalytical and statistical methods are used to estimate the pharmacokinetic parameters to achieve precision in the final results. Joint efforts are being undertaken by researchers and regulatory authorities to develop more structured systems which will pave a path towards advancement in this sector. In addition, this review can serve as an introductory disquisition for the reader to get acquainted with clinical aspects of drug development and even help him/her to choose a prospective career in this field.

\section{ACKNOWLEDGEMENT}

Authors are grateful to Dr. Neel Lahoti, MD, Synergen Bio. Pvt. Ltd., Pune for providing the necessary facility and guidance to carry out the internship work.

\section{CONFLICT OF INTEREST}

The authors declare that there is no conflict of interest.

\section{REFERENCES}

1. Sharma A, Sharma J, Rajak H, Kumar D. Joshi dr RK, Dixit V. Clinical trials in India: A scientific approach for new drug development. Curr. Res Pharm Sci. 2013;1:1-6.

2. Genest J. Clinical research. CMAJ. 1990;143(5):383-7.

3. What are the different types of clinical research? [internet]. FDA. FDA; 2018 [cited Aug 4 2021]. Available from: https://www.fda.gov/patients/clinical-trialswhat-patients-need-know/what-are-different-types-clinical-research.

4. Chew BH. Planning and conducting clinical research: the whole process. Cureus. 2019 Feb 20;11(2):e4112. doi: 10.7759/cureus.4112, PMID 31058006.

5. The Drugs Enquiry Committee. India, 1930. Indian Med Gaz. 1930;65(11):640-2.

6. Bhatt A. Evolution of clinical research: A history before and beyond James Lind. Perspect Clin Res. 2010;1(1):6-10. PMID 21829774.

7. The drugs and cosmetics act, ACT [cited Aug 4 2021]. Available from: https:// legislative.gov.in/sites/default/files/A1940-23.pdf. Vol. 23 OF Vol. 1940.pdf [Internet].

8. Drugs and cosmetics Act1940 Rules1945.pdf [internet] [cited Aug 4 2021]. Available from: https://cdsco.gov.in/opencms/export/sites/CDSCO WEB/Pdfdocuments/acts_rules/2016DrugsandCosmeticsAct1940Rules1945.pdf.

9. DCGI, Central Drugs Standard control organization, home [internet] [cited Aug 4 2021]. Available from: https://cdsco.gov.in/opencms/opencms/en/Home/.

10. DTAB DCC [internet] [cited Aug 4 2021]. Available from: https://cdsco.gov.in/ opencms/opencms/en/dcc-dtab-committee.

11. Differding E. The Drug Discovery and Development Industry in India-Two Decades of Proprietary Small-Molecule R\&D. ChemMedChem. 2017;12(11):786-818. doi: 10.1002/cmdc.201700043, PMID 28464443.

12. Imran M, Najmi AK, Rashid MF, Tabrez S, Shah MA. Clinical research regulation in India-history, development, initiatives, challenges and controversies: still long way to go. J Pharm Bioallied Sci. 2013;5(1):2-9. doi: 10.4103/0975-7406. 106553, PMID 23559817.

13. Schedule Y(amended version) - CDSCO. Vol. 48.

14. Thatte UM, Marathe PA. Ethics Committees in India: past, present and future. Perspect Clin Res. 2017;8(1):22-30. doi: 10.4103/2229-3485.198549, PMID 28194334.

15. Ethics Committee [internet] [cited Aug 4 2021]. Available from: https://cdsco. gov.in/opencms/opencms/en/Clinical-Trial/Ethics-Committee/.

16. Das NK, Sil A. Evolution of ethics in clinical research and ethics committee. Indian J Dermatol. 2017;62(4):373-9. doi: 10.4103/ijd.IJD_271_17, PMID 28794547.

17. ICMR. Ethical_Guidelines_2017.pdf [internet] [cited Aug 4 2021]. Available from: https://main.icmr.nic.in/sites/default/files/guidelines/ICMR_Ethical_Guidelines_2017.pdf.

18. E6. (R2)-good-clinical-practice--integrated-addendum-to-ICH-E6(R1).pdf [internet] [cited Aug 4 2021]. Available from: https://www.fda.gov/files/drugs/pub- 
lished/E6\% 28R2\%29-Good-Clinical-Practice--Integrated-Addendum-to-ICHE6\%28R1\%29.pdf.

19. Burt T, Sharma P, Dhillon S, Manchanda M, Mittal S, Trehan N. Clinical research environment in India: challenges and proposed solutions. J Clin Res Bioeth. 2014;5(6):1-8. doi: 10.4172/2155-9627.1000201, PMID 25590017.

20. Institutional Review Board/Independent Ethics Committee. (IRB/IEC) [internet] ICH GCP [cited Aug 4 2021]. Available from: https://ichgcp.net/news/3-institutional-review-boardindependent-ethics-committee-irbiec.

21. Nagadurga DH. Bioavailability and bioequivalence studies [internet]. IntechOpen; 2019. Pharmaceutical formulation design - recent practices [cited Aug 4 2021] Available from: https://www.intechopen.com/chapters/66222.

22. Bioequivalence of generic drugs | Psychiatrist.com [internet] [cited Aug 4 2021]. Available from: https://www.psychiatrist.com/jcp/psychopharmacology/ bioequivalence-generic-drugs/.

23. Laco M. Bioequivalence and its evaluation. Bioequiv Availab. 2021;1:13(2): No: 1000422.

24. Khandave SS, Sawant SV, Joshi SS, Bansal YK, Kadam SS. Comparative bioequivalence studies of tramadol hydrochloride sustained-release 200-mg tablets. Drug Des Dev Ther. 2010;4:367-74. doi: 10.2147/DDDT.S15133, PMID 21151623.

25. Merck Sharp and Dohme Corp. An Open-Label, Randomized, 2-Period Crossover Study to Evaluate the bioequivalence After Administration of a losartan 100-mg/ hydrochlorothiazide 12.5-mg Combination Tablet and the Coadministration of a Currently Marketed COZAARTM 100-mg Tablet and MICROZIDETM 12.5-mg Capsule in Healthy Adults [internet]; 2016 Jan. clinicaltrials.gov [cited Aug 3 2021]. Report No. : NCT00953680. Available from: https://clinicaltrials.gov/ct2/ show/NCT00953680.

26. Pfizer. Phase 1, open-label, randomized, single-dose, 4-treatment, 4-period crossover bioequivalence study comparing $25 \mathrm{Mg}$ and $50-\mathrm{Mg}$ formulations of DVS-233 SR and investigate food effect on 50-Mg formulations of DVS-233 SR tablet under fed and fasted conditions [internet]; 2011 Dec. clinicaltrials.gov [cited Aug 3 2021]. Report No. : NCT01190514. Available from: https://clinicaltrials. gov/ct2/show/NCT01190514.

27. Informed consent for clinical trials [internet]. FDA. FDA; 2019 [cited Aug 4 2021]. Available from: https://www.fda.gov/patients/clinical-trials-what-patients-needknow/informed-consent-clinical-trials.

28. Guidelines for Bioavailability and bioequivalence studies. Delhi, March: CDSCO; 2005 [internet] [cited Aug 4 2021]. Available from: https://dineshthakur.com/ wp-content/uploads/2016/06/be-guidelines-draft-ver10-march-16-05.pdf.

29. Schultz A, Saville BR, Marsh JA, Snelling TL. An introduction to clinical trial design Paediatr Respir Rev. 2019;32:30-5. doi: 10.1016/j.prrv.2019.06.002, PMID

\section{9}

30. Machin D, Fayers PM, Tai BC. Randomised clinical trials: design, practice and reporting. John Wiley \& Sons; 2021. 553 p.

31. Bellary S, Krishnankutty B, Latha MS. Basics of case report form designing in clinical research. Perspect Clin Res. 2014;5(4):159-66. doi: 10.4103/22293485.140555, PMID 25276625

32. Inspecting clinical trials - the trial master file - MHRA Inspectorate [internet] [cited Aug 4 2021]. Available from: https://mhrainspectorate.blog.gov.uk/ 2015/07/30/inspecting-clinical-trials-the-trial-master-file/.

33. Essential documents for the conduct of a clinical trial [internet]. ICH GCP [cited Aug 4 2021]. Available from: https://ichgcp.net/news/8-essential-documentsfor-the-conduct-of-a-clinical-trial.

34. Guideline on the content, management and archiving of the clinical trial master file. Vol. 17.

35. Multidisciplinary approach to protocol writing [internet]. Applied Clinical Trials [cited Aug 4 2021]. Available from: https://www.appliedclinicaltrialsonline.com/ view/multidisciplinary-approach-protocol-writing

36. Al-Jundi A, Sakka S. Protocol writing in clinical research. J Clin Diagn Res 2016;10(11):ZE10-3. doi: 10.7860/JCDR/2016/21426.8865, PMID 28050522.

37. Protocol development [internet] [cited Aug 4 2021]. Available from: https:// www.uth.edu/ctrc/trial-conduct/protocol-development.htm.

38. (PDF) Bioanalytical method development and validation: critical concepts and strategies [internet] [cited Aug 4 2021]. Available from: https://www.researchgate.net/publication/308392096_Bioanalytical_method_development_and_validation_Critical_concepts_and_strategies.

39. Han $D$, Chen $C$, Zhang $C$, Zhang $Y$, Tang $X$. Determination of mangiferin in rat plasma by liquid-liquid extraction with UPLC-MS/MS. J Pharm Biomed Anal. 2010;51(1):260-3. doi: 10.1016/j.jpba.2009.07.021, PMID 19699046.

40. What will clinical trials look like in 10 years? Experts forecast 3 key trends [internet] [cited Aug 4 2021]. Available from: https://www.clinicalleader.com/ doc/what-will-clinical-trials-look-like-in-years-experts-forecast-key-trends-0001.

41. Nass SJ, Moses HL, Mendelsohn J. Program I of M (US) C on CCT and the NCG. US: National Academies Press; 2010. Overview of conclusions and recommendations [internet]. A National Cancer Clinical Trials System for the 21st Century: Reinvigorating the $\mathrm{NCl}$ Cooperative Group Program [cited Aug 4 2021]. Available from: https://www.ncbi.nlm.nih.gov/books/NBK220382/.

Article History: Submission Date : 13-08-2021; Revised Date : 29-08-2021; Acceptance Date : $18-09-2021$.

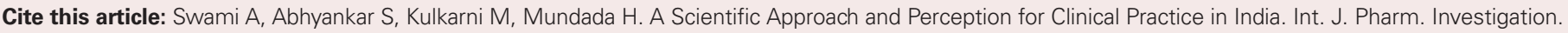
$2021 ; 11(3): 248-53$ 